\title{
NRF2 controls proteostasis through the transcriptional regulation of autophagy.
}

\author{
Marta Pajares ${ }^{1,2,3}$, Ana I Rojo ${ }^{1,2,3}$ and Antonio Cuadrado ${ }^{1,2,3}$. \\ ${ }^{1}$ Centro de Investigación en red en enfermedades Neurodegenerativas (Ciberned). \\ ${ }^{2}$ Instituto de Investigación Sanitaria La Paz (IdiPaz). \\ ${ }^{3}$ Instituto de investigaciones Biomédicas "Albertos Sols". Departamento de Bioquímica de la Facultad de Medicina de la Universidad Autónoma de \\ Madrid, (UAM). \\ * Corresponding author: \\ Ana I Rojo: airojo@iib.uam.es, Antonio Cuadrado: antonio.cuadrado@uam.es. \\ Centro de Investigación en red en enfermedades Neurodegenerativas (Ciberned). Instituto de Investigación Sanitaria La Paz (IdiPaz). Instituto de \\ investigaciones Biomédicas "Albertos Sols". Departamento de Bioquímica de la Facultad de Medicina de la Universidad Autónoma de Madrid, (UAM). \\ Madrid, Spain.
}

Introduction: Cells control the abundance and quality of the proteome through a wide network that integrates signaling pathways, gene expression and protein degradation systems. Degradation of cytosolic components inside lysosomes is carried out by specific types of autophagy in mammals, including macroautophagy and chaperone mediated autophagy (CMA). Considering autophagy as a proteostatic and defensive mechanism, we sought to determine if this process could be regulated by the transcription factor NRF2, classically considered the master regulator of the antioxidant cell response.

Material and methods: We performed a bioinformatics analysis to identify putative NRF2 binding sequences, termed antioxidant response elements (AREs) among autophagy related genes. Some of them were further validated by ChIP analysis. Messenger RNA and protein levels of cells under basal levels or submitted to different treatments were assessed by quantitative PCR and immunoblot. CMA activity in intact cells was determined with a photoswitchable reporter. The impact of NRF2 deficiency in vivo was determined by immunofluorescence and immunoblot.

Results: The bioinformatics analysis allowed us to identify putative NRF2 binding sequences, termed antioxidant response elements (AREs), in many genes whose products participate in macroautophagy and CMA. Several were further validated as NRF2-regulated genes by ChIP assays and quantitative PCR in Nrf2-deficient cells. Interestingly, Nrf2-knockout cells exhibited impaired macroautophagy flux in response to oxidative stress. Nrf2-knockout cells also showed reduced LAMP2A lysosomal levels (the limiting step for CMA) and, consequently, impaired CMA activity. Treatment with pro-oxidants up-regulated CMA through the transcriptional induction of Lamp2a, but to a lesser extent in Nrf2-deficient cells. Interestingly, pharmacological activation of NRF2 led to increased expression of many of these autophagy-related genes. This novel role of NRF2 in the regulation of autophagy may have an impact on proteinopathies, such as Alzheimer's Disease (AD). Indeed, NRF2 deficiency results in reduced neuronal expression of some of these markers as well as increased intracellular APP/A $\beta$ and insoluble TAU in a mouse model of AD.

Conclusion: Our results point to a novel role of NRF2 in the regulation of autophagy and suggest a new strategy to combat proteinopathies.

Keywords: NRF2, autophagy, oxidative stress.

Published May 18, 2018.

Copyright: (c) 2018 Authors. This is an open-access article distributed under the terms of the Creative Commons Attribution License, which permits unrestricted use, distribution, and reproduction in any medium, provided the original author and source are credited. Editor: Name of the editor here.

Cite as: Marta Pajares, Ana I Rojo, Antonio Cuadrado. NRF2 controls proteostasis through the transcriptional regulation of autophagy. IBJ Plus 2018 (S2):e00011 doi: 10.24217/2531-0151.18v1s2.00011.

Funding: FPI UAM, SAF2016-76520-R.

Competing Interests: the authors disclose no conflicts of interest. 